\title{
THE EFFECTS OF MAGNETIC NONLINEARITIES ON A STORED PROTON BEAM AND THEIR IMPLICATIONS FOR SUPERCONDUCTING STORAGE RINGS $\dagger$
}

\author{
M. CORNACCHIA \\ Lawrence Berkeley Laboratory \\ University of California \\ Berkeley, CA 94720 \\ L. EVANS \\ CERN, 1211 Geneva 23, Switzerland
}

(Received March 7, 1985)

\section{INTRODUCTION}

The topic of the effects of magnetic-field imperfections on the stability of proton beams $\ddagger$ in accelerators and storage rings is as old as the ideas of these machines. For accelerators operating in the pulsed modes (for instance, the AGS, PS, SPS, and Fermilab main ring), the order of the magnetic multipolar imperfections which have an effect on the beam dynamics is low, not higher than the 4th (decapole). This is because the fast cycle time prevents a sizable beam blowup during acceleration and extraction due to high-order multipolar imperfections. In colliding-beam facilities like the ISR or the SPS $p-\bar{p}$, the freedom in the choice of working point allows one to keep away from low-order resonances, since this choice is not dictated by slow extraction considerations. On the other hand, there is no experimental evidence of the effects of high-order magnetic-imperfection resonances§ in these machines, because of the high field quality achievable with conventional magnets. $\|$

In superconducting magnets the field quality is determined by, amongst other factors, the accuracy in the positioning of the superconducting coil blocks, rather than by the iron shape (as in conventional magnets). Moreover, for a large storage ring like the SSC, there is a strong incentive to keep the magnet aperture as small as possible in order to reduce cost. For these reasons, high-order multipolar magnetic imperfections play a greater role than they do in machines like the ISR or the SPS $p-\bar{p}$. How great their role is, is not yet known, and the

† This work was supported by the Director, Office of Energy Research, Office of High Energy and Nuclear Physics, High Energy Physics Division, U.S. Dept. of Energy, under Contract No. DE-AC03-76SF00098 and under Brookhaven National Laboratory DOE Contract No. DE-AC02$76 \mathrm{CH} 00016$.

¥We consider here and in what follows the stability of proton beams only. For electrons other considerations, like the sextupolar correction for storage rings and radiation damping, come into play. $\S$ In what follows, we define high-order resonances as those of order higher than $5(5 v=p)$.

$\|$ High-order resonances have been observed in the ISR attributable to the presence of the high- $\beta$ superconducting quadrupole, ${ }^{1}$ but experimental evidence is rather limited. 
motivation for these experiments and others, which may follow, is to shed some light on the beam dynamics and on the theoretical models.

It is generally accepted that high-order nonlinear resonances alone do not pose a threat to the stability of the beam, because the nonlinear detuning, always present in a real dynamic situation, prevents indefinite build-up of amplitudes. In order to have beam diffusion, some time-dependent factors have to be introduced, such as repeated crossing of a resonance due to synchrotron oscillations or power-supply ripple. Moreover, in order to have growth, the betatron phase must be random at each crossing. If it is not, the particle amplitude is modulated and no growth occurs unless the unperturbed tune lies on a synchro-betatron resonance.

A randomizing process has been proposed ${ }^{2,3}$ consisting of nonlinear de-tuning and a low modulation frequency. This model predicts that a low frequency is more harmful than a high frequency, because of the appearance of stochasticity when synchro-betatron resonances overlap.

In this report we describe an experiment which was conducted at the SPS to observe the combined effects of a single isolated high-order nonlinear resonance, nonlinear detuning, and tune modulation. The nonlinear resonance, as well as the nonlinear detuning, was driven by a nonlinear lens, which had been previously used at the SPS for the study of the beam-beam interactions. ${ }^{4}$ The nonlinear motion of a particle in the field of the lens is discussed.

\section{BEAM DYNAMICS WITH A NONLINEAR LENS}

\section{A. Resonance Width and Nonlinear Detuning}

The nonlinear lens installed in the SPS is similar to the one built at the ISR to simulate the beam-beam effects. ${ }^{5}$ It consists of a pair of current-carrying copper bars which can be positioned above and below the beam to produce a very nonlinear magnetic field.

The magnetic-field components can be obtained from Ampere's law. With the nomenclature of Fig. 1, they are

$$
\begin{aligned}
& B_{x}(x, y)=\frac{\mu_{o} I y}{\pi} \frac{x^{2}+y^{2}-h^{2}}{\left[x^{2}+(h+y)^{2}\right]\left[x^{2}+(h-y)^{2}\right]}, \\
& B_{y}(x, y)=-\frac{\mu_{o} I x}{\pi} \frac{x^{2}+y^{2}+h^{2}}{\left[x^{2}+(h+y)^{2}\right]\left[x^{2}+(h-y)^{2}\right]} .
\end{aligned}
$$

Here, $I$ is the lens current, and $h$ is the half distance of the axis of the conductors.

The quadrupole gradient for small amplitudes and the linear tune shift $\Delta v_{L}$ (horizontal or vertical) are then

$$
\begin{aligned}
\frac{\partial B_{y}}{\partial x} & =-\frac{\partial B_{x}}{\partial y}=-\frac{\mu_{o} I}{\pi h^{2}}, \\
\Delta v_{L} & =\frac{\beta_{x, y}}{4 \pi} \frac{L}{B \rho} \frac{\mu_{o} I}{\pi h^{2}},
\end{aligned}
$$




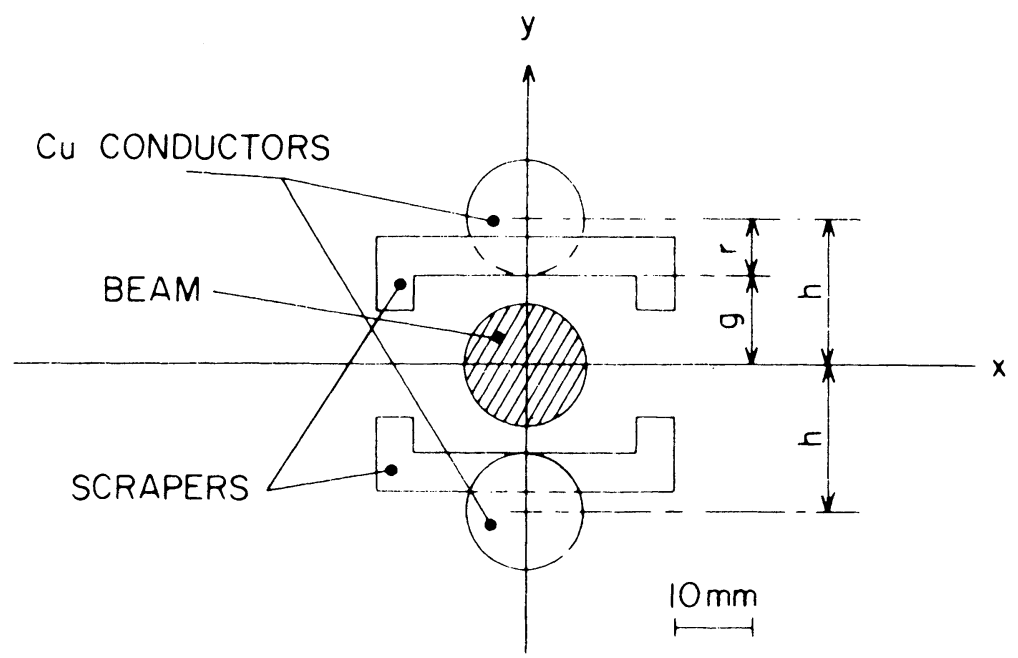

FIGURE 1 View of nonlinear lens.

respectively. $L$ is the bar length, $\beta_{x, y}$ is the horizontal or vertical $\beta$-function values at the bar location, and $B \rho$ is the magnetic rigidity.

We consider the one-dimensional motion in the vertical plane

$$
\frac{d^{2} y}{d s^{2}}+K(s) y=\frac{B_{x}(y)}{B \rho} f\left(s_{o}\right),
$$

where the lens is a short perturbation of length $L$ at azimuth $s_{o}$.

Transformation into Courant and Snyder variables gives

$$
\frac{d^{2} \eta}{d \theta^{2}}+v^{2} \eta=v^{2} \beta^{3 / 2} g(\eta) f(\theta)
$$

where

$$
\begin{aligned}
\eta & =y / \sqrt{\beta}, \frac{d \theta}{d s}=\frac{1}{v \beta}, \\
g(\eta) & =\frac{4 \pi \Delta v_{L}}{L \beta^{1 / 2}} \eta_{h}^{2} \frac{\left(\eta+\eta_{d}\right)}{\eta_{h}^{2}-\left(\eta-\eta_{d}\right)^{2}},
\end{aligned}
$$

where $\eta_{h}^{2}=h^{2} / \beta, \eta_{d}=d / \sqrt{\beta}$, and $d$ is the lens displacement with respect to the beam axis. If the lens is localized over a small distance $L$, the azimuthal dependence is

$$
f(\theta)=\frac{L}{\pi v \beta}\left[\frac{1}{2}+\sum_{p=1}^{\infty} \cos (p \theta)\right] .
$$

The Hamiltonian of Eq. (4) is

$$
H\left(\eta, \eta^{\prime}, \theta\right)=\frac{1}{2} \eta^{\prime 2}+\frac{1}{2} v^{2} \eta^{2}+v^{2} \beta^{3 / 2} g_{1}(\eta) f(\theta),
$$


with

$$
g_{1}(\eta)=-\frac{1}{2} \ln \left|\eta_{h}^{2}-\left(\eta-\eta_{d}\right)^{2}\right| \frac{4 \pi \Delta v_{L}}{L \beta^{1 / 2}} \eta_{h}^{2}
$$

We transform, as is customary, to the new variables $\varepsilon, \phi$ via the transformation

$$
\eta=-\sqrt{\frac{2 \varepsilon}{v}} \cos \phi ; \quad \eta^{\prime}=\sqrt{2 v \varepsilon} \sin \phi
$$

and the new Hamiltonian becomes

$$
\hat{H}=v \varepsilon+2 \Delta v_{L} v \eta_{h}^{2} \ln \left|\eta_{h}^{2}-\left(\sqrt{\frac{2 \varepsilon}{v}} \cos \phi+\eta_{d}\right)^{2}\right| \cdot\left[\frac{1}{2}+\sum_{m=1}^{\infty} \cos (m \theta)\right] .
$$

The phase equation is, after some manipulations and introducing the transverse emittance $E_{T}=2 \varepsilon / v$,

$$
\frac{d \phi}{d \theta}=\frac{\partial \hat{H}}{\partial \varepsilon}=v-\frac{4 \Delta v_{L} \cos \phi(\cos \phi+\delta)}{1-\alpha^{2}(\cos \phi+\delta)^{2}}\left[\frac{1}{2}+\sum_{p=1}^{\infty} \cos (p \theta)\right]
$$

where

$$
\alpha=\sqrt{\varepsilon \beta} / h, \quad \delta=d / \sqrt{\varepsilon \beta} .
$$

The amplitude-dependent tune shift $\Delta v_{N L}$ can be obtained by averaging the zeroth harmonic component of Eq. (10) over a period

$$
\frac{\Delta v_{N L}}{\Delta v_{L}}=\frac{1}{\pi} \int_{0}^{2 \pi} \frac{\cos \phi(\cos \phi+\delta) d \phi}{1-\alpha^{2}(\cos \phi+\delta)^{2}}
$$

The resonance width $\Delta v_{n}$ is obtained by transforming to the "slow" phase $\psi=\phi-p \theta / n$ and averaging the coefficient of the slowly varying term $\cos n \psi$ :

$$
\frac{\Delta v_{n}}{\Delta v_{L}}=\frac{2}{\pi} \int_{0}^{2 \pi} \frac{\cos \phi(\cos \phi+\delta) \cos (n \phi)}{1-\alpha^{2}(\cos \phi+\delta)^{2}} d \phi
$$

This expression automatically takes into account the effect of all the multipole components. A similar analysis in the horizontal plane gives the same formulas as Eqs. (11) and (12), except for a positive sign in front of the $\alpha^{2}(\cos \phi+\delta)^{2}$ term.

Equations (11) and (12) have been integrated numerically. $\dagger$

Figure 2 shows the nonlinear detuning $\Delta v_{N L} / \Delta v_{L}$ as a function of the normalized amplitude $\alpha=\sqrt{\varepsilon \beta} / h$. The dashed curve was obtained by considering only the octupole component of the lens field:

$$
\frac{\Delta v_{N L}}{\Delta v_{L}}=\frac{3 \alpha^{2}}{4} \text {. }
$$

The higher multipoles have a very strong effect in the vertical plane at large amplitude, especially for a misaligned lens.

†They can, in fact, be integrated exactly, as has been shown by E. Courant ${ }^{3}$. 


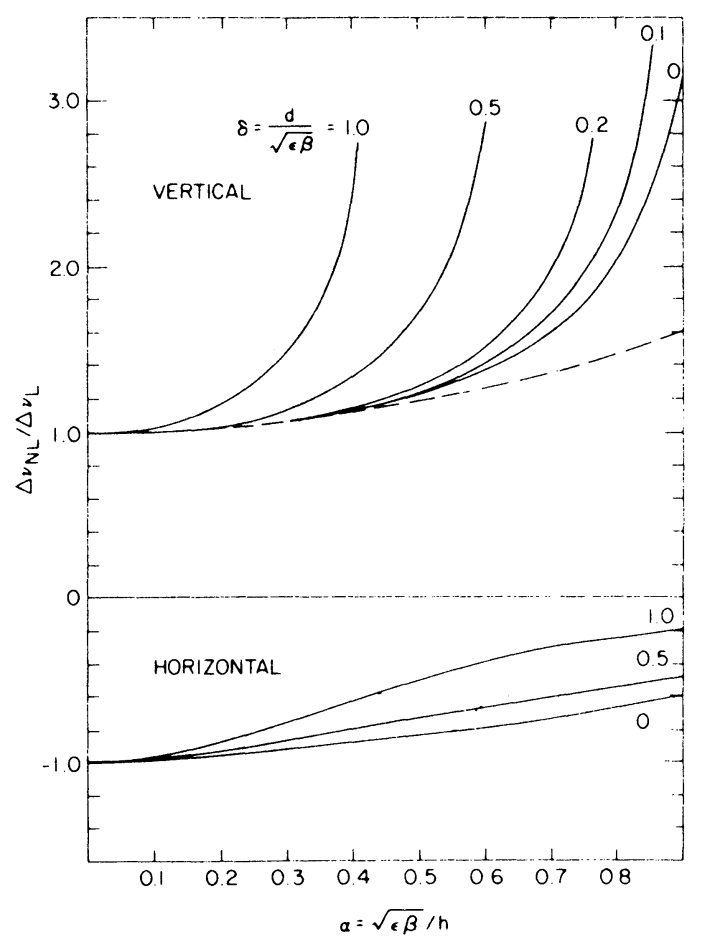

FIGURE 2 Detuning effect of nonlinear lens as a function of the normalized amplitude, $\alpha$.

For a perfectly aligned lens $(\delta=0)$, only even-order resonances are excited. The resonance width computed by isolating only the lowest multipole is simply

$$
\frac{\Delta v_{n}}{\Delta v_{L}}=\left(\frac{\alpha}{2}\right)^{n-2}
$$

The resonance widths at large amplitude are stronger than expected from this formula in the vertical plane, as would be expected from the increase in field strength with amplitude. On the contrary, they are considerably weaker in the horizontal plane.

\section{B. Island Width and Tune Modulation}

Let us introduce the slow phase $\psi=-\phi+p \theta / n$ in the Hamiltonian Eq. (9). Let us write the new Hamiltonian, in absence of tune modulation and in the vicinity of a resonance of order $n$, as

$$
\bar{H}=\left(v-\frac{p}{n}\right) \varepsilon+U(\varepsilon)+v \eta_{h}^{2} V_{n}(\varepsilon) \cos (n \psi) .
$$

In this form, $v \eta_{h}^{2} d V_{n}(\varepsilon) / d \varepsilon$ is the resonance width, and $d U(\varepsilon) / d \varepsilon$ is the nonlinear detuning. Both have already been defined. 
$V_{n}(\varepsilon)$ is obtained by averaging the coefficient of the slowly varying term in the Hamiltonian:

$$
\begin{aligned}
V_{n}(\varepsilon) & =\frac{\Delta v_{L}}{\pi} \int_{0}^{2 \pi} \cos (n \phi) \ln \left|1-\frac{2 \varepsilon}{v \eta_{h}^{2}}\left(\cos \phi+\eta_{d} \sqrt{\frac{v}{2 \varepsilon}}\right)^{2}\right| d \phi \\
& =\frac{\Delta v_{L}}{\pi} \int_{0}^{2 \pi} \cos (n \phi) \ln \left|1-\alpha^{2}(\cos \phi+\delta)^{2}\right| d \phi .
\end{aligned}
$$

Stable islands occur at $\partial \bar{H} / \partial \varepsilon=0, \partial \bar{H} / \partial \psi=0$ :

$$
\left\{\begin{array}{l}
(v-p / n)+\frac{d U(\varepsilon)}{d \varepsilon}+v \eta_{h}^{2} \frac{d V_{n}(\varepsilon)}{d \varepsilon} \cos (n \psi)=0, \\
n V_{n}(\varepsilon) \sin (n \psi)=0 .
\end{array}\right.
$$

The island width is [6]

$$
\Delta \varepsilon=4 \sqrt{\frac{V_{n}(\varepsilon) v \eta_{h}^{2}}{d^{2} U / d \varepsilon^{2}}}
$$

In the presence of tune modulation, the resonance at $v=p / n$ is split into sidebands separated by $\Delta v=v_{s} / n$ and reduced in strength by the factor $J_{m}\left(n \Delta \hat{v} / v_{s}\right)$, where $v_{s}$ is the synchrotron tune, $\Delta \hat{v}$ the peak modulation amplitude, and $m$ the order of the satellite.

The separation of these islands in the variable $\varepsilon$ is

$$
\Delta \varepsilon=\frac{v_{s}}{n d^{2} U / d \varepsilon^{2}},
$$

and their width is

$$
\Delta \varepsilon=4 \sqrt{\frac{V_{n}(\varepsilon) J_{n}\left(\hat{n} \Delta v / v_{s}\right) v \eta_{h}^{2}}{d^{2} U / d \varepsilon^{2}}} .
$$

The stochastic limit is achieved when the islands' separation is equal to or smaller than the islands' width:

$$
\frac{v_{s}}{n d^{2} U / d \varepsilon^{2}} \leq 4 \sqrt{\frac{V_{n}(\varepsilon) J_{n}\left(n \Delta \hat{v} / v_{s}\right) v \eta_{h}^{2}}{d^{2} U / d \varepsilon^{2}}},
$$

where $d^{2} U / d \varepsilon^{2}$ is the derivative of the nonlinear detuning function $D(\varepsilon)=d U /$ $d \varepsilon$; this can be written in terms of the amplitude $\alpha$ as $d^{2} U / d \varepsilon^{2}=D^{\prime}(\alpha) /$ $v \eta_{h}^{2} \alpha ; V_{n}(\varepsilon)$ is given in Eq. (16). In terms of the parameter $\alpha$, Eq. (21) becomes

$$
v_{s} \leq\left[\frac{16 n D^{\prime}(\alpha) V_{n}(\alpha)}{\alpha} \sqrt{\frac{n}{\pi \Delta \hat{v}}}\right]^{2 / 3},
$$

with

$$
\begin{aligned}
& D^{\prime}(\alpha)=\frac{2 \Delta v_{L} \alpha}{\pi} \int_{0}^{2 \pi} \frac{\cos \phi(\cos \phi+\delta)^{3}}{\left[1-\alpha^{2}(\cos \phi+\delta)^{2}\right]^{2}} d \phi, \\
& V_{n}(\alpha)=\frac{\Delta v_{L}}{\pi} \int_{0}^{2 \pi} \cos (n \phi) \ln \left|1-\alpha^{2}(\cos \phi+\delta)^{2}\right| d \phi .
\end{aligned}
$$

The integrals of Eq. (23) have been tabulated numerically. 


\section{NONLINEAR-LENS AND SUPERCONDUCTING-MAGNET FIELD ERRORS}

Coil-positioning errors are the main sources of magnetic imperfections in superconducting magnets. The high-order multipoles in these magnets are much stronger than in conventional magnets, and there is some concern over the long-term stability of the beam in the presence of magnetic imperfections in superconducting storage rings.

We discuss the analogy between these imperfections and the field created by the nonlinear lens. We suggest that such a nonlinear lens may be used in existing conventional accelerators (like the SPS or the FNAL accelerator) to test the stability of the beam in superconducting accelerators and the validity of the tolerance criterion which will be adopted for the coil-positioning errors.

For coil-dominated superconducting magnets with the coil block placed within the iron shield, and with blocks arranged in a $\cos \phi$ current distribution, the result for the rms-error multipoles at the magnet center, neglecting the iron shield, has been derived, and a rough form of this result is, for the main dipoles,

$$
\left(\Delta b_{n}^{\mathrm{rms}}\right)_{s c}=\left(\frac{2}{N_{b}}\right)^{1 / 2} \frac{(n+1)\langle\delta\rangle}{R_{c}^{n+1-k}} .
$$

Here

$N_{b}=$ number of blocks,

$R_{c}=$ mean coil radius,

$\langle\delta\rangle=$ rms tolerance in coil positioning,

$k=0$ for dipoles, and

$k=1$ for quadrupoles.

The multipolar term $\Delta b_{n}$ is defined as $\Delta B=B_{o} \Delta b_{n} x^{n}$, where $\Delta B$ is the field at the displacement $x$ from the center along the median plane. $B_{o}$ is the main bending field, or, in the case of quadrupoles, the field gradient.

The nonlinear coefficients of the nonlinear lens are ${ }^{4}$

$$
\left(\Delta b_{n}\right)_{\text {lens }}=\frac{\mu_{o} I}{\pi h^{n+1} B_{o}} .
$$

We now assume that the stop-band width excited by a single multipole coefficient is a measure of the strength of the nonlinearity. For the superconducting dipoles in the SSC we have, for the rms value of the one-dimensional stop-band width,

$$
\begin{aligned}
\left\langle\Delta v_{\mathrm{SSC}}(N)\right\rangle= & \frac{F(N)}{2^{N-2}} \frac{1}{4 \pi \rho_{\mathrm{sSC}}}\left(\varepsilon_{\mathrm{SSC}} \bar{\beta}_{\mathrm{SSC}}\right)^{(N-2) / 2} \bar{\beta}_{\mathrm{SSC}} L_{M} \sqrt{M} \\
& \times\left(\frac{2}{N_{b}}\right)^{1 / 2} \frac{N\langle\delta\rangle}{R_{c}^{N}} .
\end{aligned}
$$

Similarly, for the nonlinear lens in the SPS,

$$
\Delta v_{\mathrm{SPS}}(N)=\frac{F(N)}{2^{N-2}} \frac{1}{4 \pi\left(B_{o} \rho\right)_{\mathrm{SPS}}}\left(\varepsilon_{\mathrm{SPS}} \beta_{\mathrm{SPS}}\right)^{(N-2) / 2} \beta_{\mathrm{SPS}} \frac{\mu_{o} I}{\pi h^{N}} L_{l},
$$


where

$N=$ order of the resonance,

$F(N)=1.6$ for $N=3$ or $4 ; F(N)=1$ for all other $N$,

$M=$ number of magnets contributing to the driving term of the resonance,

$\rho=$ bending radius,

$\varepsilon=$ horizontal or vertical emittances,

$\bar{\beta}_{\text {SSC }}=$ average $\beta$ function in the magnets,

$L_{M}=$ magnet length in the SSC, and

$L_{l}=$ length of nonlinear lens in the SPS.

Equation (26) assumes that the resonance is driven primarily by one set of dipoles, e.g., the dipole near a focusing quadrupole. If the quadrupoles are the more important contributors to the resonance, then the formula should be modified by the factor $R_{c} G / B_{o}$, where $G$ is the quadrupole gradient. Also, in Eq. (26) we use the approximation $\left(\sum_{i} \beta_{i}^{N}\right) \sim M \bar{\beta}^{N}$, which is clearly not correct. However, for high-order resonances, only the high- $\beta$ perturbations contribute significantly to the width of the stop bands, and the approximation becomes more valid. In any case, we are only interested in a scaling law. The stop-band widths quoted further on have been computed with the correct formula, which takes into account all the elements in the ring, each with its own value of the $\beta$ function.

The ratio of the stop-band widths in SSC and in the SPS is thus

$$
\frac{\left\langle\Delta v_{\mathrm{SSC}}(N)\right\rangle}{\Delta v_{\mathrm{SPS}}(N)}=C N\left(\frac{h}{R_{C}}\right)^{N}\left(\frac{\sigma_{\mathrm{SSC}}}{\sigma_{\mathrm{SPS}}}\right)^{N-2},
$$

where

$$
C=\frac{\bar{\beta}_{\mathrm{SSC}}}{\beta_{\mathrm{SPS}}} \frac{\rho_{\mathrm{SPS}}}{\rho_{\mathrm{SSC}}} \frac{L_{M} \sqrt{M}}{L_{l} \mu_{o} I}\left(\frac{2}{N_{b}}\right)^{1 / 2}\langle\delta\rangle \pi B_{o \mathrm{SPS}},
$$

and $\sigma$ is the betatron beam size in the magnet or the lens locations.

For perfect analogy, the term

$$
N\left(\frac{h}{R_{c}}\right)^{N}\left(\frac{\sigma_{\mathrm{SSC}}}{\sigma_{\mathrm{SPS}}}\right)^{N-2}
$$

should be constant for all $N$ 's. Evidently, this condition cannot be satisfied, but a set of parameters can be found which, for a resonance up to a certain order, gives stop-band widths of the same magnitude.

As an example, we give the stop-band widths computed according to Eq. (12) and with the following lens parameters:

$$
\begin{aligned}
L & =1.5 \mathrm{~m}, \\
I_{\max } & =2000 \mathrm{~A}, \\
\beta_{H} & =25 \mathrm{~m}, \text { and } \\
\beta_{V} & =87.5 \mathrm{~m} .
\end{aligned}
$$

The three parameters $\alpha, \delta$, and $I$ can be chosen to fit resonance-width profiles as well as possible.

As a rough example, we consider some estimated random stop-band imperfections for a particular version of the SSC lattice. ${ }^{8,9}$ The stop-band half-widths for 
TABLE I

Comparison of Stop-Band Half-Widths: Nonlinear Lens (NLL) Experiment: Predicted Values for the SSC

\begin{tabular}{ccccc}
\hline $\begin{array}{c}\mathrm{N} \\
\begin{array}{c}\text { Order of the } \\
\text { resonance }\end{array}\end{array}$ & $\begin{array}{c}\text { Horizontal } \\
\text { width, } \\
\text { NLL }\end{array}$ & $\begin{array}{c}\text { half- } \\
\text { Vertical } \\
\text { half-width, } \\
\text { NLL }\end{array}$ & $\begin{array}{c}\text { Horizontal and } \\
\text { vertical half- } \\
\text { widths; SSC } \\
\text { with nominal } \\
\text { emittance }\end{array}$ & $\begin{array}{c}\text { Horizontal and } \\
\text { vert. half-width; } \\
\text { SSC with } 3 \mathrm{~mm} \\
\text { maximum amplitude } \\
\text { in regular cells }\end{array}$ \\
\hline 3 & $8.2 \times 10^{-5}$ & $1.0 \times 10^{-4}$ & $6.3 \times 10^{-4}$ & $3.3 \times 10^{-3}$ \\
4 & $6.9 \times 10^{-5}$ & $8.8 \times 10^{-5}$ & $2.8 \times 10^{-6}$ & $7.6 \times 10^{-5}$ \\
5 & $2.1 \times 10^{-6}$ & $2.9 \times 10^{-6}$ & $4.1 \times 10^{-8}$ & $5.7 \times 10^{-6}$ \\
6 & $1.0 \times 10^{-6}$ & $1.5 \times 10^{-6}$ & $2.0 \times 10^{-10}$ & $1.5 \times 10^{-7}$ \\
7 & $4.3 \times 10^{-8}$ & $6.6 \times 10^{-8}$ & $2.4 \times 10^{-12}$ & $9.9 \times 10^{-9}$ \\
\hline
\end{tabular}

the SSC refer to the cases of a nominal emittance of $10^{-8} \pi$ m-rad at $1 \mathrm{TeV}$ and of a maximum amplitude of $3 \mathrm{~mm}$ in the regular cells. With $\alpha=0.15, \delta=0.5$, we get the results of Table I. The stop bands are calculated for the unidimensional resonances $N v=P$, where $v$ is the horizontal or vertical tune.

\section{DESCRIPTION OF EXPERIMENTAL CONDITIONS}

The SPS was set up with injection at $26 \mathrm{GeV}$. The lattice was the normal lattice used for fixed-target physics (no low $\beta$ 's). The beam was accelerated to $270 \mathrm{GeV}$, and an indefinitely long flat top was manually set up for a good pulse. The beam current at $270 \mathrm{GeV}$ was low, about $5 \times 10^{11} \mathrm{ppp}$. Each pulse consisted of a fast extracted CPS pulse filling one eleventh of the SPS circumference. The low beam current was desirable to avoid confusion by collective instabilities. The intensity was, however, high enough to obtain a good signal on the Schottky monitors.

After optimizing the beam in the ramp, the chromaticities were measured in the flat top by displacing the orbit with the rf and measuring the tunes. The measured values were $C_{H}=C_{V}=3.7$. The chromaticities $C_{H, V}$ are defined as $\Delta v / \Delta P / P$.

Next, the momentum spread of the debunched beam was calculated by measuring the width of the longitudinal Schottky line:

$$
\Delta f_{\mathrm{rev}}=n f_{\mathrm{rev}} \eta \Delta P / P \text {. }
$$

The spread in revolution frequencies $\Delta f_{\text {rev }}$ is shown in Fig. $3 ; n$ is a multiple of the revolution frequency, equal to 247 in our case; $\eta=1.8 \times 10^{-3} ; f_{\text {rev }}=$ revolution frequency $=43375 \mathrm{~Hz}$. The line width at the base is about $75 \mathrm{~Hz}$, giving

$$
\Delta P / P= \pm 1.9 \times 10^{-3} .
$$

The core of the beam is only half the above value. The tune spread from the 


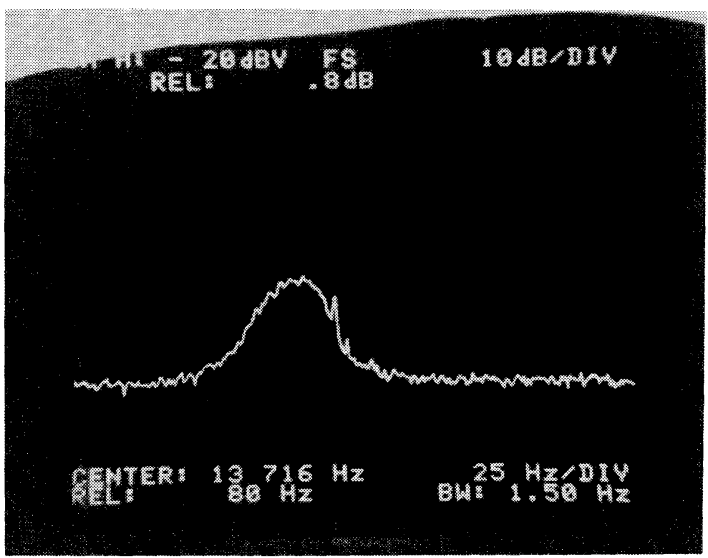

FIGURE 3 Longitudinal Schottky scan of a debunched beam, giving the spread in revolution frequencies.

measured chromaticities is then

$$
\Delta v_{H, V}= \pm 3.7 \times 1.9 \times 10^{-3}= \pm 7.0 \times 10^{-3}
$$

at the base of the distribution, and half this value at the core.

These values of the tune spread can be checked by measuring the width of the transverse Schottky lines (Fig. 4):

$$
\Delta f_{\beta}=(n \pm v) \eta f_{\mathrm{rev}} \frac{\Delta P}{P} \pm f_{\mathrm{rev}} C \Delta P / P .
$$

$\Delta P / P$ is known from the longitudinal measurement, and $C$ is the chromaticity (horizontal or vertical).

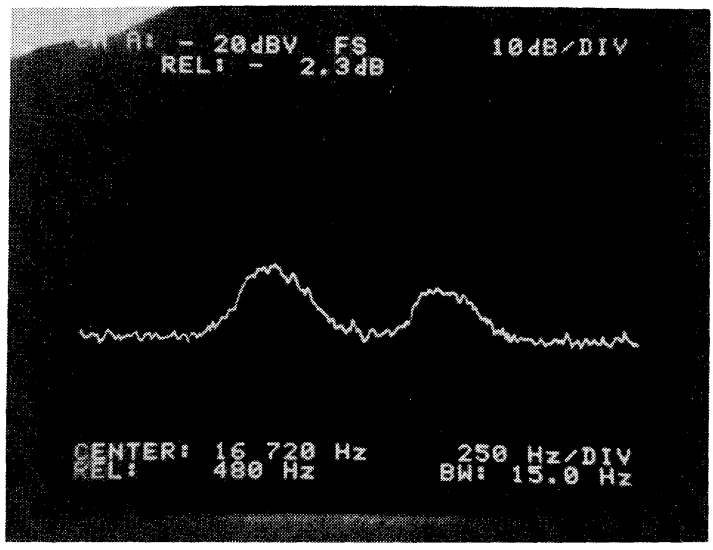

FIGURE 4 Transverse Schottky scan of a debunched beam. The lower-frequency trace is the vertical scan. 


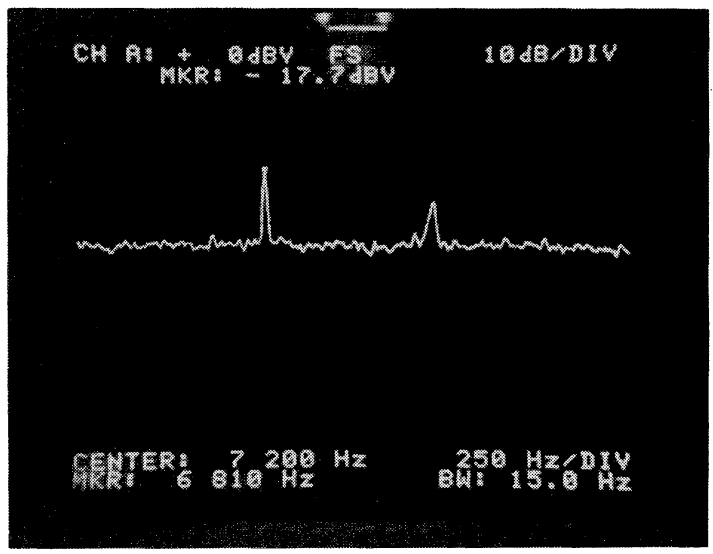

FIGURE 5 Transverse Schottky scan of a bunched beam. The lower-frequency trace is the horizontal scan.

The slow wave [minus sign in Eq. (32)] is recognizable because the width of the corresponding line is wider above transition for positive chromaticity.

The tunes were measured with the transverse Schottky scan of a bunched beam (Fig. 5):

$$
\begin{aligned}
& v_{H}=26.6230, \\
& v_{V}=26.6007 .
\end{aligned}
$$

The tunes could be measured with an accuracy of $2 \times 10^{-4}$ in tune, and, during the long flat top (up to one hour long), we observed no appreciable shift in tune at the peak of the transverse bunched Schottky scan. The width of the lines of Fig. 5 is due to the nonlinear detuning and to a power-supply ripple. The nonlinear detuning of the machine is very small, since the octupoles were not energized. The only other source of nonlinear detuning is the sextupoles; this is a second-order effect, and the detuning is estimated to be less than $10^{-4}$ in tune. The SPS power supply, on the other hand, is known to suffer from a random jitter which changes approximately every second. The amplitude of this jitter is estimated to be $\sim \pm 5 \times 10^{-4}$ in tune, and this is probably responsible for the widths of the lines of Fig. 5.

\section{RESULTS. FIFTH-ORDER RESONANCE STUDIES}

The purpose of the experiment was to study the effect of the 5th-order resonance $5 v_{y}=133$ when crossed repeatedly at various frequencies and amplitudes of the tune modulation. This resonance is excited by the nonlinear lens having the following characteristics (unless otherwise specified):

$h=37.2 \mathrm{~mm}$,

$$
I \text { (lens current })=1500 \mathrm{~A} \text {, }
$$


$\Delta v_{L}=0.005$,

$\alpha=0.25$,

$\delta=0.20$ (the lens is displaced vertically by $1.9 \mathrm{~mm}$ with respect to the beam axis),

$\beta_{H}$ (at lens $)=87.5 \mathrm{~m}$,

$\beta_{V}$ (at lens) $=25 \mathrm{~m}$,

$E_{T}$ (beam emittance) $\left.=1 \pi \times 10^{-6} \mathrm{~m}-\mathrm{rad}\right)$.

The vertical-beam emittance of $1 \pi \times 10^{-6} \mathrm{~m}$-rad is obtained in a controlled way by restricting the aperture to this value with scrapers and by blowing up the beam until some losses are observed. The beam was blown up by driving the plates normally used as dampers with an rf signal sweeping through the first $(n-v)$ line at around $17.6 \mathrm{kHz}$ repetitively until some beam loss was observed.

The calculated half-width of the $5 v_{y}=133$ resonance with the above lens parameters is

$$
\Delta v_{e}=2.9 \times 10^{-6}
$$

This is 70 times the value corresponding to the SSC estimate at $1 \mathrm{TeV}$. It is also the value pertinent to the case of a maximum betatron amplitude of $2.4 \mathrm{~mm}$ in the regular cells (see Table 1 ).

The nonlinear lens-induced vertical tune spread is

$$
\Delta v_{N L}=2.3 \times 10^{-4} \text {. }
$$

The linear tune spread, discussed in the previous paragraph, is

$$
\Delta v_{L} \sim \pm 7.0 \times 10^{-3} \text {, }
$$

including the tails of the beam. This spread is approximately the same for bunched and unbunched beams, since the rf was switched off nonadiabatically.

The maximum closed-orbit distortion introduced by the lens being displaced is only $0.03 \mathrm{~mm}$.

In what follows we describe various experimental situations. Note that each time a parameter is changed and an observation made, the beam is blown up transversely until it touches the scrapers. This guarantees the reproducibility of the beam size and the associated parameters, such as resonance width and nonlinear detuning. The scrapers were left in their aperture-limiting positions during the experiment. Thus, the beam losses or lifetime reduction refer to particles whose vertical "emittance" exceeds $1 \pi \times 10^{-6} \mathrm{~m}$-rad.

a. The lens is off. The $\mathrm{rf}$ is on. The synchrotron-oscillation frequency is $200 \mathrm{~Hz}$. The working line is shown in Fig. 6. The tail of the tune distribution touches the coupling resonance $4 v_{V}+v_{H}=133$, but this resonance is not excited by the vertically displaced lens.

The beam lifetime is $\sim 20$ hours.

b. The lens is excited. The vertical tune shifts by 0.005 and is brought back to the resonance (Fig. 7).

The rf is still on. For a tune-modulation amplitude $\Delta \hat{v}_{V}=3.5 \times 10^{-3}$, the frequency-threshold condition is, from Eq. (22), $7.6 \mathrm{~Hz}$ for $\alpha=0.25$. Thus, since the synchrotron-oscillation frequency is $200 \mathrm{~Hz}$, the local Chirikov criterion 


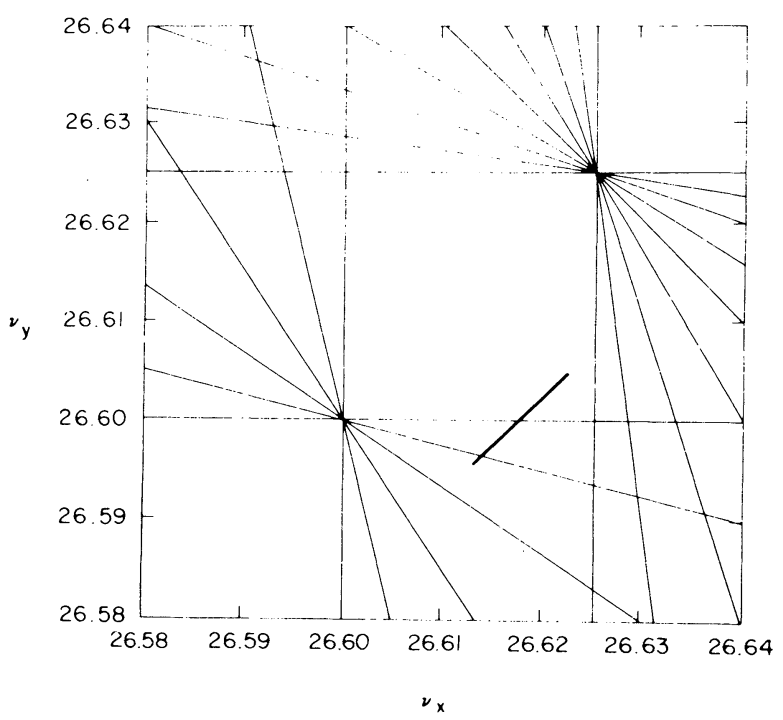

FIGURE 6 Working line (case a). The tune diagram contains all sum resonances up to and including the 8 th order.

predicts no growth for particles having the largest betatron amplitudes in the distribution.

The lifetime is still $\sim 20$ hours. The nonlinear lens seems to have no effect on the bunched beam.

c. The rf is switched off and the beam is coasting. The lens is on. No change in lifetime (still $\sim 20$ hours) or in the transverse Schottky-scan distribution is observed.

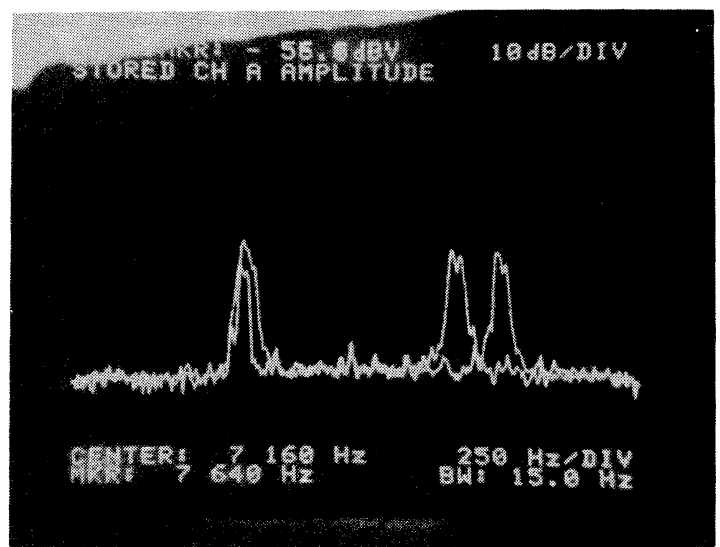

FIGURE 7 Transverse Schottky scans of a bunched beam before and after the excitation of the nonlinear lens. 
The above three experiments showed no effects on the beam when the resonance is crossed at a frequency of $200 \mathrm{~Hz}$ with a tune-modulation amplitude varying from zero (on momentum particles) to $7.0 \times 10^{-3}$ (particles at the tails of the momentum distribution).

In the following experiments a tune ripple was introduced by exciting the power supply of a single quadrupole with a sinusoidal oscillation. Because of the $\beta$-function values at the quadrupole location $\left(\beta_{H}=18 \mathrm{~m} ; \beta_{V}=109 \mathrm{~m}\right)$, the tune modulation is preferentially vertical. The range of frequencies allowed by the power-supply characteristics and by the response of the magnet is from 1 to about $40 \mathrm{~Hz}$.

d. With the lens off and the rf off, the lifetime is high ( $\sim 20$ hours) and insensitive to the frequency (in the range $1 \div 40 \mathrm{~Hz}$ ) and amplitude [in the range $\left(3 \times 10^{-4}\right) \div\left(3 \times 10^{-3}\right)$ ] of the tune modulation. Thus we see no effect due to the natural 5th-order resonance of the machine.

e. The lens is switched on. The amplitude of the tune modulation is $\Delta \hat{v}=3 \times 10^{-4}$. The $\mathrm{rf}$ is off. The theoretical frequency threshold for the local stochastic criterion is $17 \mathrm{~Hz}$. We drive the tune modulation with a frequency of $1 \mathrm{~Hz}$; thus, we should be well in the stochastic regime.

The lifetime drops from 20 to 10 hours. After 1 hour of storage the lifetime is still around 10 hours, but we start to see some changes in the tune distribution (Fig. 8). The dips in distribution correspond to $v_{H}=26.6178, v_{V}=26.6000$. The distribution shows a depletion of particles lying on the $5 v_{V}=133$ resonance; these particles also have $v_{H} \sim 26.6178$, and a hole is formed in the horizontal tune distribution corresponding to this frequency.

f. We increase the tune-modulation frequency to $5 \mathrm{~Hz}$. The $\mathrm{rf}$ is off. The lens is on. The amplitude of the vertical tune modulation is still $3 \times 10^{-4}$.

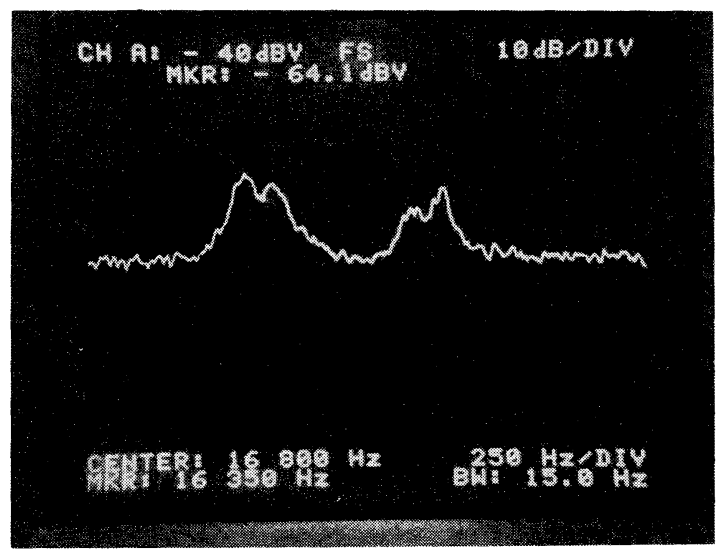

FIGURE 8 Transverse Schottky scans of a debunched beam. The lower-frequency trace is the vertical scan, which shows a dip in the distribution corresponding to $v_{V}=26.6000$. 


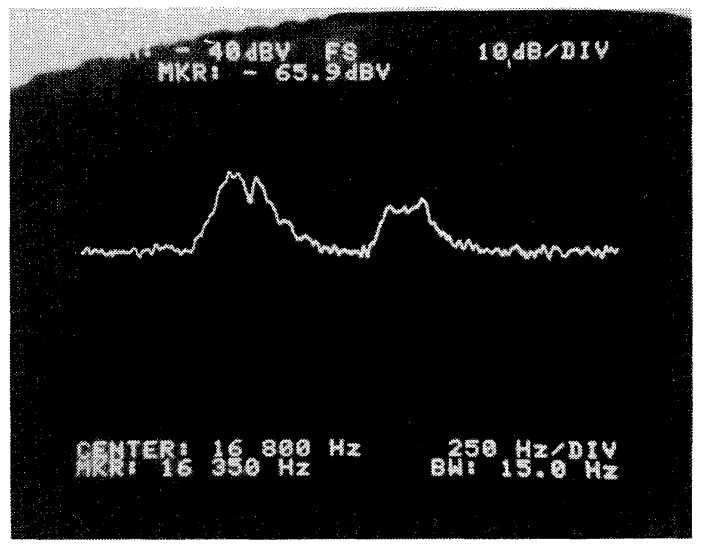

FIGURE 9 Transverse Schottky scans of a debunched beam. The depletion of particles becomes more evident.

The lifetime is still 10 hours. The depletion of particles becomes more evident (Fig. 9).

g. We increase the vertical tune-modulation amplitude to $\Delta \hat{v}=3 \times 10^{-3}$. The rf is off. The lens is on. The tune-modulation frequency is $1 \mathrm{~Hz}$, still below the theoretical threshold for these conditions $(8 \mathrm{~Hz})$. The tune distribution becomes flat, since the tune modulation covers the whole core of the beam (Fig. 10). The lifetime drops to 2 hours.

h. We increase the tune-modulation frequency to 10,20 , and $40 \mathrm{~Hz}$, keeping

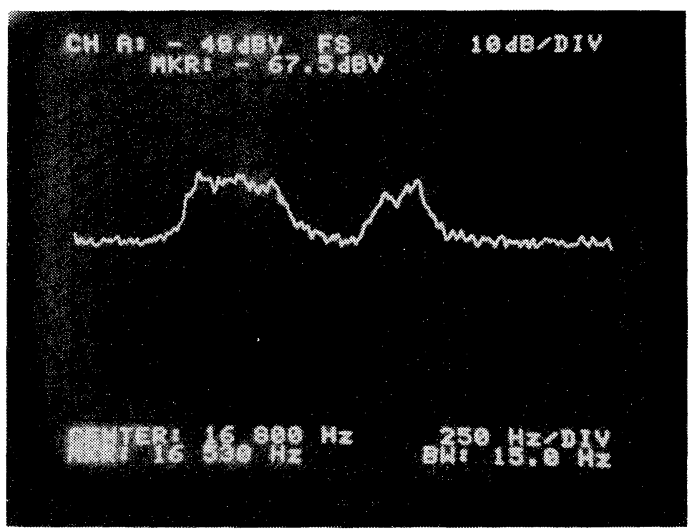

FIGURE 10 Transverse Schottky scans of a debunched beam. The lower-frequency trace is the vertical scan. The tune modulation covers the whole core of the beam, causing the distribution to flatten. 


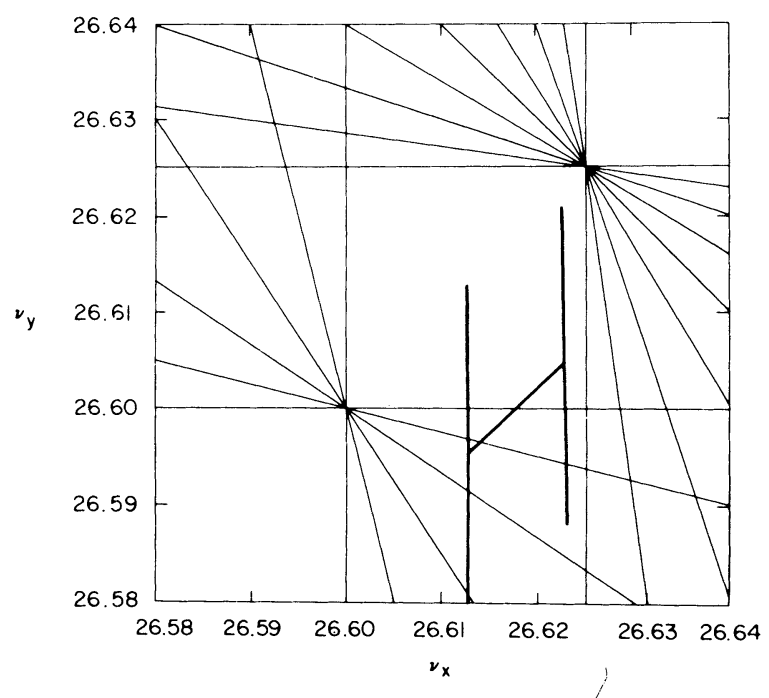

FIGURE 11 Tune diagram showing the excursion of the working line when the amplitude of the tune modulation is increased to 0.017 . The diagram contains sum-resonance lines up to and including the 8th order.

the amplitude at $\Delta \hat{v}=3 \times 10^{-3}$. The rf is off; the lens is on. There is a tendency for the lifetime to increase with increasing frequency from 2 hours (with $1-\mathrm{Hz}$ modulation) to 4 hours (40- $\mathrm{Hz}$ modulation).

i. The amplitude of the vertical tune modulation is increased to $\Delta \hat{v}=0.017$, with a frequency of $1 \mathrm{~Hz}$. The rf is off; the lens is on. The particles now cross three 5th-order resonances, but only two $\left(5 v_{H}=p, 3 v_{V}+2 v_{H}=p\right)$ are driven by the vertically displaced lens (Fig. 11). The lifetime is still about 2 hours, not very different from the case with the much smaller modulation amplitude $\Delta \hat{v}=0.003$. Evidently, the effect of the additional resonance which has to be crossed is offset by the faster speed of crossing.

$\mathrm{j}$. The strength of the lens is halved, $\Delta v_{L}=0.0025$. The $\mathrm{rf}$ is off. The vertical tune-modulation amplitude is $\Delta \hat{v}=3 \times 10^{-3}$; the frequency is $1 \mathrm{~Hz}$.

The lifetime is 4 hours, indicating a linear scaling law with the lens strength (see case $g$ ). Figure 12 shows the transverse and longitudinal Schottky scans taken about $15 \mathrm{~min}$ after the excitation of the lens. The longitudinal scan indicates a clear loss of particles corresponding to the large hole in the momentum distribution.

$\mathrm{k}$. The rf is switched on; the quadrupole modulation is turned off. The horizontal and vertical chromaticities are increased, by changing the sextupole settings, to $C_{H}=C_{V}=27$. This corresponds to a tune spread (including the tails of the beam distribution) of $\Delta v_{H, V}= \pm 0.05$ (Fig. 13).

With lens off, the beam lifetime is about 2 hours.

With lens on, the beam lifetime is about 1.5 hours. 

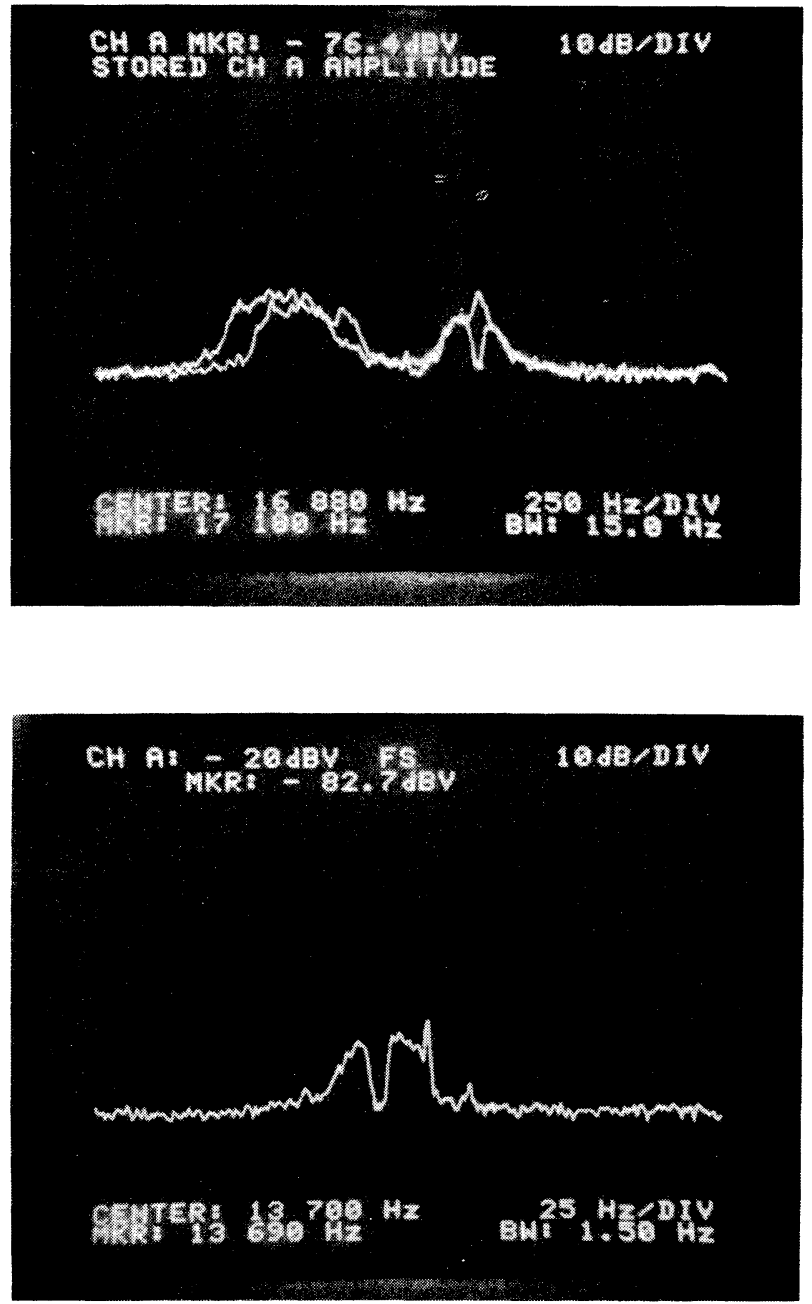

FIGURE 12 Transverse (top photograph) and longitudinal Schottky scans. The longitudinal scan shows loss of particles corresponding to the hole in the distribution.

These results indicate that the natural machine resonances (in particular, we suspect, the proximity of the third-integer resonance $3 v_{H}=3 v_{V}=68$ ) are responsible for the loss in beam lifetime, and that the lens contributes only marginally to the low lifetime.

The results of the experiments described above are summarized in Table II.

\section{RESULTS. EIGHTH-ORDER RESONANCE STUDIES}

An experiment similar in procedure to the one just described was conducted for the 8 th-order resonance $8 v_{V}=213$. This resonance can be driven by the lens 


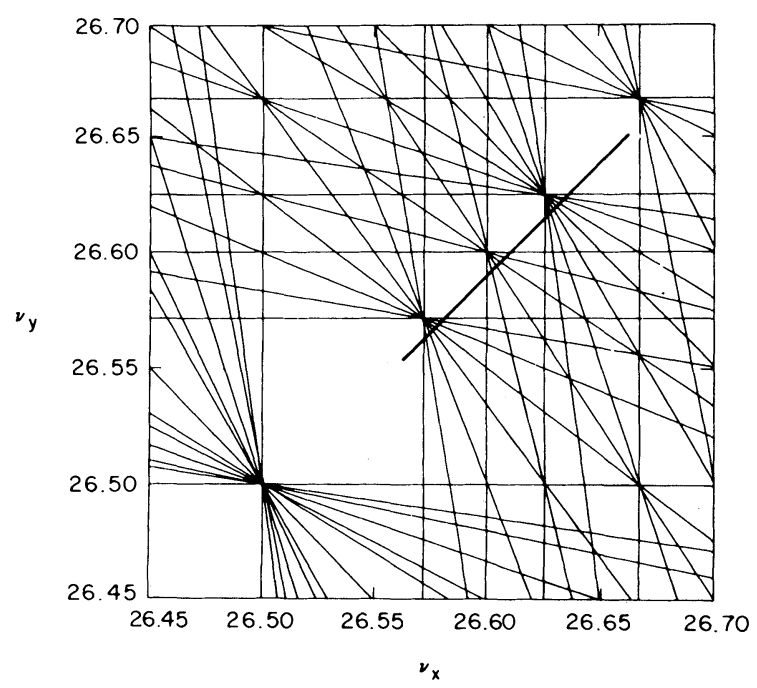

FIGURE 13 Tune diagram showing the working line for case $k$. The diagram contains sumresonance lines up to and including the 8 th order.

centered on the beam. The following conditions were applied:

$$
\begin{aligned}
v_{H} & =26.6400, \\
v_{V} & =26.6250 \\
\Delta v_{L} & =0.01 \\
\alpha & =0.5 \\
E_{T} & =1 \pi \times 10^{-6} \mathrm{~m}-\mathrm{rad} \\
\Delta v_{H, V} & = \pm 2 \times 10^{-3} \\
\Delta v_{N L} & =2.4 \times 10^{-3}, \text { and } \\
\Delta v_{e} & =4.9 \times 10^{-6}
\end{aligned}
$$

\begin{tabular}{|c|c|c|c|c|c|c|c|}
\hline case & $\mathrm{rf}$ & $\begin{array}{l}\text { Tune } \\
\text { Spread } \\
( \pm)\end{array}$ & Lens & $\Delta v_{L}$ & $\begin{array}{c}\text { Tune- } \\
\text { modulation } \\
\text { frequency } \\
(\mathrm{Hz})\end{array}$ & $\begin{array}{l}\text { Tune- } \\
\text { modulation } \\
\text { amplitude }\end{array}$ & $\begin{array}{l}\text { Lifetime } \\
\text { (h) }\end{array}$ \\
\hline a. & ON & 0.007 & OFF & - & - & 0 & 20 \\
\hline b. & ON & 0.007 & ON & 0.005 & - & 0 & 20 \\
\hline c. & OFF & 0.007 & ON & 0.005 & - & 0 & 20 \\
\hline $\begin{array}{l}\text { d. } \\
\text { e. }\end{array}$ & OFF & 0.007 & OFF & - & $1 \div 40$ & $\left.\begin{array}{l}3 \times 10^{-4} \\
3 \times 10^{-3}\end{array}\right\}$ & 20 \\
\hline f. & OFF & 0.007 & ON & 0.005 & 1 & $3 \times 10^{-4}$ & 10 \\
\hline g. & OFF & 0.007 & ON & 0.005 & 5 & $3 \times 10^{-4}$ & 10 \\
\hline h. & OFF & 0.007 & ON & 0.005 & 1 & $3 \times 10^{-3}$ & 2 \\
\hline i. & OFF & 0.007 & ON & 0.005 & $20 \div 40$ & $3 \times 10^{-3}$ & 4 \\
\hline j. & OFF & 0.007 & ON & 0.005 & 1 & 0.017 & 2 \\
\hline k. & OFF & 0.007 & ON & 0.0025 & 1 & $3 \times 10^{-3}$ & 4 \\
\hline \multirow[t]{2}{*}{1.} & ON & 0.05 & OFF & - & - & - & 2 \\
\hline & ON & 0.05 & ON & 0.005 & - & - & 1.5 \\
\hline
\end{tabular}

TABLE II

Effect of various parameters on beam lifetime 
TABLE III

Effect of various parameters on beam lifetime

\begin{tabular}{|c|c|c|c|c|c|c|}
\hline rf & $\begin{array}{c}\text { Tune } \\
\text { spread } \\
( \pm)\end{array}$ & Lens & $\Delta v_{L}$ & $\begin{array}{c}\text { Tune- } \\
\text { modulation } \\
\text { frequency } \\
(\mathrm{Hz})\end{array}$ & $\begin{array}{c}\text { Tune- } \\
\text { modulation } \\
\text { amplitude }\end{array}$ & $\begin{array}{l}\text { Lifetime } \\
\text { (h) }\end{array}$ \\
\hline OFF & $2 \times 10^{-3}$ & OFF & - & - & 0 & 30 \\
\hline ON & $2 \times 10^{-3}$ & OFF & - & - & 0 & 30 \\
\hline ON & $2 \times 10^{-3}$ & ON & 0.01 & - & 0 & 30 \\
\hline OFF & $2 \times 10^{-3}$ & ON & 0.01 & - & 0 & 30 \\
\hline OFF & $2 \times 10^{-3}$ & OFF & - & 1 & $1.5 \times 10^{-3}$ & 30 \\
\hline OFF & $2 \times 10^{-3}$ & ON & 0.01 & 1 & $1.5 \times 10^{-3}$ & 2 \\
\hline
\end{tabular}

We recall that $\Delta v_{H, V}$ is the linear tune spread in the beam, $\Delta v_{L}$ the linear tune shift of the lens, $\Delta v_{N L}$ the nonlinear tune shift induced by the lens, and $\Delta v_{e}$ the resonance half-width.

The results of the experiment are summarized in Table III.

Because of machine problems, a more extensive investigation could not be carried out. The results collected so far, however, show the same behavior observed during the study of the 5th-order resonance described in Section V; the resonance is innocuous in absence of tune modulation or when crossed repeatedly at the synchrotron-oscillation frequency $(200 \mathrm{~Hz})$. A low-frequency ripple causes a reduction in the beam lifetime.

\section{DISCUSSION OF THE RESULTS}

The experimental evidence indicates that a low-frequency tune modulation and the associated crossings of nonlinear resonances cause an important reduction in beam lifetime. The threshold frequency does not come out very sharply from the experiment, although the evidence is that the lifetime doubles when going from $1-\mathrm{Hz}$ to $40-\mathrm{Hz}$ modulation. It was unfortunate that power-supply limitations prevented us from investigating frequencies above $40 \mathrm{~Hz}$. However, it was seen quite clearly that a modulation frequency of $200 \mathrm{~Hz}$ driven by synchrotron oscillations is completely harmless provided one keeps the tune away from low-order resonances. The difference between the low- and high-frequency studies is in the distribution of the tune-modulation amplitude: when the tune modulation is driven by a power-supply ripple, all the particles in the beam experience the same amplitude and frequency of tune modulation; when this modulation is caused by synchrotron oscillations, the amplitude of the modulation follows the distribution in the longitudinal phase space. The frequency varies little with the maximum momentum deviation, since the rf bucket was only partially filled. The experiments have shown a clear dependence of the beam lifetime on the modulation frequency, although, in the range of frequencies accessible to us, no sharp threshold could be detected. Nevertheless, we believe that the following 
broad conclusions can be drawn:

- High-order nonlinear resonances are harmless in absence of tune modulation.

- Fast crossing of the resonances induced by synchrotron oscillations are much less harmful than slow crossing of the resonances, driven by power-supply ripple.

\section{CONCLUSIONS}

A nonlinear lens may be used to study the effect of high-order multipolar-field imperfections on a stored proton beam. Such a nonlinear lens is particularly suitable to simulate field imperfections of the types encountered in coildominated superconducting magnets. We have studied experimentally at the SPS the effect of high-order (5th and 8th) single isolated resonances driven by the nonlinear lens. The width of these resonances is of the order one expects to be caused by field errors in superconducting magnets of the SSC type. The experiment shows that, in absence of tune modulation, these resonances are harmless. Slow crossings of the resonance, on the other hand, have destructive effects on the beam, much more so than fast crossings caused by synchrotron oscillations.

We suggest that, in the design of future storage rings, sources of low-frequency tune modulation be avoided as a way to reduce the harmful effects of high-order multipolar-field imperfection.

\section{ACKNOWLEDGMENTS}

We would like to thank W. Kalbreier for building the lens and, together with $\mathrm{H}$. Kuhn and S. Peggs, for helping with measurements.

\section{REFERENCES}

1. J. Poole, private communication.

2. J. Tennyson, in Nonlinear Dynamics and the Beam-Beam Interaction, AIP Conf. Proc., 57, Brookhaven National Laboratory, (AIP, New York, 1979).

3. E. D. Courant, ISABELle Project, Tech. Note 163 (1980).

4. J. P. Delahaye, L. Evans, J. Gareyte, and W. Kalbreier, SPS Improvement Report 180 (1980).

5. E. Keil and G. Leroy, IEEE Trans. Nucl. Sci., NS-22, 1370 (1975).

6. A. Schoch, CERN Report 57-21 (1958).

7. G. Parzen, Part. Accel., 6, 237 (1975).

8. A. Garren, in Proc. 12th Int. Conf. on High-Energy Accel., FNAL, Aug. 11-16, 1983; LBL Report LBL-16023, SSC Note-1 (1983).

9. L. Smith, LBL Report LBID-811, SSC Note-8 (1983). 